Research Article

Esko K. Juuso*

\title{
Expertise and Uncertainty Processing with Nonlinear Scaling and Fuzzy Systems for Automation
}

https://doi.org/10.1515/eng-2020-0080

Received Oct 06, 2019; accepted Apr 29, 2020

\begin{abstract}
Integration of domain expertise and uncertainty processing is increasingly important in automation solutions which rely on data analytics and artificial intelligence. We need a level to assess what is approximately correct. Uncertainties of the inputs are taken into account by using fuzzy numbers as the inputs of different fuzzy and parametric systems. Nonlinear scaling functions (NSFs) integrate these solutions and make them easier to tune. Fuzzy rule-based systems are represented with scaled fuzzy inputs. Membership functions (MFs) can be developed from NSFs and existing MFs can be used in developing NSFs. Fuzzy set systems and linguistic equation $(L E)$ systems become consistent within the limits of detail. In recursive analysis, both meanings and interactions on all levels can be tuned together with genetic algorithms. In applications, the modular overall system consists of similar subsystems, which are normally used, with extensions to fuzzy. The compact fuzzy modules can be developed for specific tasks which are combined within Cyber Physical Systems (CPS). Uncertainty processing is embedded in the recursive analysis. The fuzzy extensions provide a feasible way for the sensitivity analysis of the solution.
\end{abstract}

Keywords: fuzzy set systems, fuzzy arithmetics, domain expertise, uncertainty

\section{Introduction}

Domain expertise and uncertainty processing are increasingly important in automation solutions which rely on data analytics and artificial intelligence. Keeping the appropriate operating conditions and assessing the approximate correctness are needed to avoid faulty decisions. Fuzzy systems provide tools where the uncertainties of the

\footnotetext{
*Corresponding Author: Esko K. Juuso: Control Engineering,
} University of Oulu, Finland; Email: esko.juuso@oulu.fi inputs are taken into account by using fuzzy numbers, labels and rules. Fuzzy set systems use degrees of membership for calculating firing the rules [1, 2]. Fuzzy inputs can introduce additional active labels and thus more conditions for the firing of rules. Additional alternatives of outputs with different degrees of membership increase the uncertainty of the response. Fuzzy relations are based on the intensities of interactions [3]. These methodologies are closely related.

Alternatively, fuzzy inputs can be processed with fuzzy arithmetic where the calculations use the fuzzy extensions of the interval analysis [4]. Equations and models include functions which can be handled with the extension principle [5]. The fuzzy result can be sufficient, e.g. in diagnostics, but it can be defuzzified in a normal way. The fuzzy mathematics has tools for handling inequalities which provide additional fuzzy facts for the rulebased fuzzy systems. Any fuzzy response can be further processed with the fuzzy arithmetic instead of going to the defuzzification.

Adaptation, which is essential in varying operating conditions, is an important part of fuzzy systems: scaling, modifying membership functions and updating rules are supported in many ways. There are many mechanisms for self-organising [6] and self-tuning [7] fuzzy controllers. Powering modifiers can be used for the fuzzy numbers and labels [8].

More or less automatic modelling possibilities have increased the popularity of artificial neural networks (ANNs) in building nonlinear transformation layers for complicated interactions within different sources of varying data [9]. Self-organising maps (SOM), which have many alternatives for calculating distances in the competitive layer [10], are suitable for clustering and shaping fuzzy rule-based systems. SOM is a feasible solution for adaptive filtering [11]. The fuzzy Kohonen clustering network integrates the fuzzy c-means (FCM) model into the Kohonen network [12].

Linguistic equation $(L E)$ systems originate from fuzzy systems [13]: nonlinear scaling makes the solutions compact by facilitating the use of linear methodologies for

ə Open Access. (c) 2020 E. K. Juuso, published by De Gruyter. (cc) BY

This work is licensed under the Creative Commons Attribution 4.0 License 
analysing interactions [14]. The systems are parametric and can be tuned with genetic algorithms [15], also recursively [16]. The compact solution makes them suitable for big data analysis and Cyber Physical Systems (CPS) [17].

This paper analyses alternatives of the fuzzy extensions of parametric systems. Meanings of variables, different types fuzzy and parametric systems are building blocks of these systems (Section 2). Feasible adaptation solutions are presented in Section 3 and application requirements for modelling, control and diagnostics in Section 4. The compact systems for varying operating conditions are discussed in Section 5. Conclusions and future work are presented in Section 6.

\section{Methodologies}

Statistical models are in various ways used in data-driven modelling and rule-based systems form the basis for handling expertise. Both lines of modelling can be efficiently extended to nonlinear applications by modifying the variables to correspond better with the meanings of the variable levels. Then the linear models and rulebases can focus on the directions of interactions.

\subsection{Meanings}

In fuzzy systems, the labels are represented by membership functions $(M F s)$ which provide the link to the numeric values. Scaling and modifying the parameters of the membership functions are efficient in the adaptation of the fuzzy systems. Nonlinear scaling functions (NSFs) make the sets of MFs consistent [14].

\subsubsection{Membership functions}

In fuzzy set theory, a fuzzy subset $A$ on universe (domain) $E$ is defined by representing the characteristic function $c_{A}$ by a membership function $\mu(x) \in[0,1]$.

In the vertical representation, a fuzzy set $A=$ $\left\{\left(u, \mu_{A}(u) \mid u \in \mathcal{U}\right\}\right.$ can be defined by a sum of ordered pairs: membership degree/set element:

$$
A=\mu_{A}\left(u_{1}\right) / u_{1}+\cdots+\mu_{A}\left(u_{n}\right) / u_{n}=\sum_{i=1}^{n} \mu_{A}\left(u_{i}\right) / u_{i},
$$

where + means $a / u+b / u=\max (a, b) / u$. This can be extended to discrete and continuous. A fuzzy set can be pre- sented in the form of two related vectors:

$$
\begin{aligned}
x & =\left[x_{1}, x_{2}, \ldots, x_{n}\right]^{T}, \\
\mu & =\left[\mu_{A}\left(x_{1}\right), \mu_{A}\left(x_{2}\right), \ldots \mu_{A}\left(x_{n}\right)\right]^{T} .
\end{aligned}
$$

Vertical MFs are used together with rule-based systems (Section 2.2.1) and relation-based systems (Section 2.2.2).

Horisontal MFs are represented by $\alpha$-cuts $A_{\alpha}$ :

$$
A_{\alpha}=\left\{x \mid x \in E \text { and } \mu_{A} \geq \alpha\right\},
$$

where $\alpha \in(0,1]$. The $\alpha$-cut operator is also denoted by $\alpha$-cut $(A, \alpha)$. The $\alpha$-cuts of higher membership degrees are always subsets of the $\alpha$-cuts of lower membership degrees:

$$
\alpha \geq \alpha \prime \Rightarrow A_{\alpha} \subseteq A_{\alpha^{\prime}} \text { and } A_{\alpha}=\lim _{\beta \uparrow \alpha} A_{\beta} .
$$

Horisontal MFs are used in fuzzy arithmetic (Section 2.2.3).

A fuzzy number $A$ is a fuzzy set of the real line with a normal, (fuzzy) convex and continuous membership function of bounded support. Fuzzy numbers are defined by support and core: $\operatorname{supp}(A)=\left\{x \mid \mu_{A}(x)>0\right\}$, and $\operatorname{core}(A)=\left\{x \mid \mu_{A}(x)=1\right\}$. The height is defined by supremum: the smallest degree of membership that is greater than or equal to each of the set. The representation can be either vertical or horisontal.

$M F s$ are normally presented with mathematical functions, e.g. triangular, trapezoidal and bell-shaped functions. Also piece-wise linear functions are used. Fuzzy rulebased systems use sets of MFs. In developing $M F s$, probability distributions can be used by interpreting MFs on the basis of possibilistic extension.

Fuzzy modifiers provide intensifying or weakening adverbs to obtain new terms:

$$
A_{1} \subseteq A_{2} \subseteq A_{3} \subseteq A_{4} \subseteq A_{5},
$$

which correspond to the powers of the membership in the powering modifiers (Table 1). Using modifiers for $\alpha$ values of the $\alpha$-cuts can be a feasible alternative. Memberships can be further processed with the conjunction (and), disjunction (or) and negation (not), see more examples in [8].

Table 1: Modifiers of fuzzy numbers [18]

\begin{tabular}{llc}
\hline Fuzzy number & Fuzzy label & Degree of membership \\
\hline$A_{1}$ & extremely $A$ & $\mu^{4}$ \\
$A_{2}$ & very $A$ & $\mu^{2}$ \\
$A_{3}$ & $A$ & $\mu$ \\
$A_{4}$ & more or less $A$ & $\mu^{\frac{1}{2}}$ \\
$A_{5}$ & roughly $A$ & $\mu^{\frac{1}{4}}$ \\
\hline
\end{tabular}


Modifiers can be used for introducing additional membership functions or fuzzy numbers which take better the domain expertise into account. Modifiers can be used for both $M F s$ by changing $\mu(x)$ vectors in vertical and $\alpha$-cuts in horisontal representation, respectively.

\subsubsection{Nonlinear scaling}

The nonlinear scaling was presented already in 1991 [13, 19] as a methodology for improving membership functions of fuzzy set systems. Nonlinear scaling functions (NSFs) are monotonously increasing functions $x_{j}=f\left(X_{j}\right)$ where $x_{j}$ is the variable and $X_{j}$ the corresponding scaled variable in the range $[-2,2]$. The function $f()$ consist of two second order polynomials, one for the negative values of $X_{j} \in[-2,0]$ and one for the positive values $X_{j} \in[0,2]$, respectively. Five parameters are needed to define these functions since the overall functions are continuous (Figure 1). [14]

The core area $\left[\left(c_{l}\right)_{j},\left(c_{h}\right)_{j}\right]$, corresponding $[-1,1]$, is within the support area defined by the minimum and maximum values. The shapes are defined by

$$
\begin{aligned}
\alpha_{j}^{-} & =\frac{\left(c_{l}\right)_{j}-\min \left(x_{j}\right)}{c_{j}-\left(c_{l}\right)_{j}}, \\
\alpha_{j}^{+} & =\frac{\max \left(x_{j}\right)-\left(c_{h}\right)_{j}}{\left(c_{h}\right)_{j}-c_{j}},
\end{aligned}
$$

where $c_{j}$ is the central tendency value, corresponding 0 . The scaling functions are monotonous and increasing if these ratios are both in the range $\left[\frac{1}{3}, 3\right]$, see [15]. The parameters of the scaling functions can be defined manually or by data-based methodologies [20]. In genetic tuning, the shape requirements are taken into account. In manual tuning, the parameters are modified to achieve monotonously increasing functions.

The corresponding inverse functions $x_{j}=f^{-1}\left(X_{j}\right)$ based on square root functions are used for scaling to the scaled range.

Monotonously increasing nonlinear scaling functions, $x_{j}=f\left(X_{j}\right)$ and $x_{j}=f^{-1}\left(X_{j}\right)$, are suitable for inductive mappings in the extension principle. This can be used in transforming fuzzy numbers and membership functions to both directions between the real and scaled values.

The NSFs, also called membership definitions, originate from $M F s$ [14]. A set of $M F s$ can be generated by selecting the locations for the functions (Figure 2). Natural language interpretation provides a basis for the transformation. The integer numbers $\{-2,-1,0,1,2\}$ correspond labels \{very low, low, normal, high, very high $\}$ or \{high negative, negative, zero, positive, high positive\} or \{accelerating decrease, decrease, constant, increase, accelerating in-

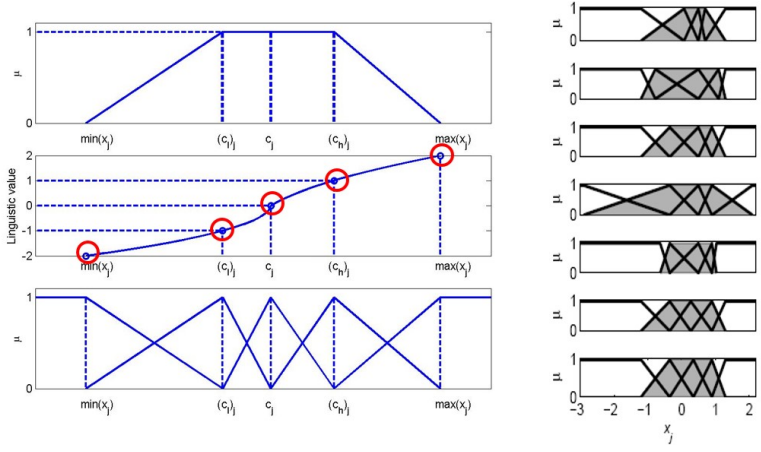

Figure 1: Nonlinear scaling and membership functions.

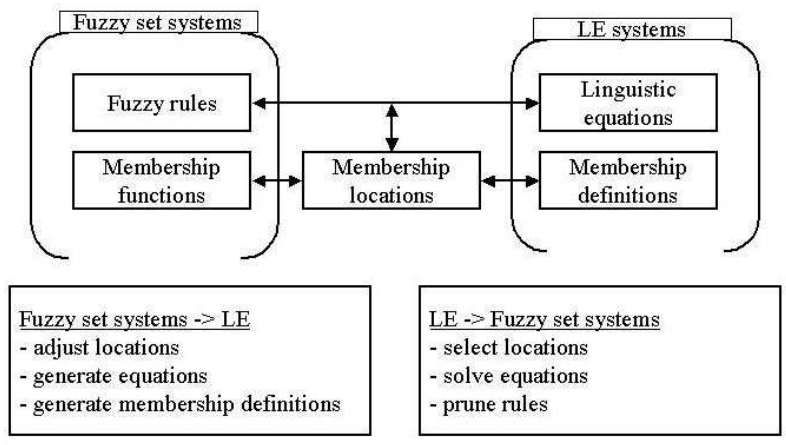

Figure 2: Fuzzy set systems and linguistic equations [14].

crease\}. The vocabulary can also be chosen in a different way, e.g. by using modifiers highly, fairly or quite [21]. Only the sequence of the labels is important.

The set of MFs depends strongly on the shape of the NSFs as can be seen in the examples shown in Figure 1. The number of $M F s$ is not limited to five. Modifiers of the labels can introduce domain expertise for defining corresponding membership locations in the scaled range (Figure 2).

The scaling can be extended to the temporal analysis: increasing and decreasing changes can be quantified, trend and fluctuation indices are calculated by using scaled values. Resulting values are within the range $[-2,2]$ and can be interpreted with MFs as in Figure 1 and explained with natural language.

Uncertainties are embedded in the nonlinear scaling approach: feasible ranges and labels are presented with $M F s$ (Figure 1). The data-based computation of the NSFS is done by using equal-sized sub-blocks, i.e. the norm for several samples can be obtained as the norm for the norms of individual samples [20,22]. The parameters can have differences between the sub-blocks, which introduces uncertainties to the NSFs and MFs. 


\subsection{Fuzzy systems}

Fuzzy systems are based on rules, relations and local models, including fuzzy numbers and labels represented by $M F s$. The uncertainties of the facts are processed by the calculations with the degrees of membership. Fuzzy systems may include versatile facts, including labels, equalities and inequalities. The nonlinear scaling improves the numerical calculations in these systems.

\subsubsection{Rule-based fuzzy set systems}

Fuzzy set systems focus on the linguistic meanings of the variables, which suit very well to qualitative descriptions of the process. In linguistic fuzzy systems, both the an tecedents and consequents are fuzzy propositions, which can be interpreted by using natural language, heuristics and common sense knowledge. The rules can have several consequents. Fuzzy rule-based systems maintain clear connections with expert systems [23]. Fuzzy set theory first presented by Zadeh [1] form a conceptual framework for linguistically represented knowledge.

Local models based on crisp functions of the antecedent variables are used in Takagi-Sugeno (TS) fuzzy models [24]. The consequent is a parameterized function, whose structure remains constant and only the parameters vary. For linear models, smoothing techniques are needed to avoid erroneous hedges and valleys on the response surfaces [25]. The freedom of parameters needs to be reduced to improve system development [26].

Type-2 fuzzy models introduced by Zadeh in 1975 take into account uncertainty about the membership function [27]. Most systems based on interval type-2 fuzzy sets are reduced to an interval-valued type-1 fuzzy set. However, the level 2 is important for the uncertainty processing. The nonlinear scaling can introduce Type-2 MFs, see Section 2.1.2.

Rule-based fuzzy set systems are highly flexible: facts can be based on fuzzification of crisp or fuzzy input values, manually provided domain expertise or calculations by using changes, trends, fluctuations, equalities and inequalities. Each fact has a degree of membership.The systems can handle contradictory data, i.e. some consequents may define forbidden areas for the outputs [28, 29].

The operating area of different types of rule-based fuzzy set systems can be extended by selecting membership locations (Figure 2) for inputs and/or outputs together with the $L E$ models are needed to tune the sets of $M F s$, see Section 2.3.3. The rulebase can focus on the directions of interactions. For example, all the alternative $M F s$ shown in
Figure 1 could be handled with the same rule-based system with an appropriate set of $M F s$.

\subsubsection{Fuzzy relational systems}

Fuzzy relational models [3] allow one particular antecedent proposition to be associated with several different consequent propositions. Each element of the relation represents the degree of association between the individual reference fuzzy sets defined in the input and output domains. The relations can be represented by fuzzy rule-based systems where the rules have weight values. In these models, the membership functions can be chosen in a fairly mechanistic way since the association intensities take care of the interactions.

These systems suit well for higher levels of abstraction where the labels are objects which do not necessarily have any connections with numeric values. NSFs could be useful in translating the intensities $[0,1]$ to the range $[-2,2]$ and natural language.

\subsubsection{Fuzzy arithmetic}

Fuzzy arithmetic provides methods for including uncertainty processing within equations and models. The $e x$ tension principle is the basic generalization of the arithmetic operations if the inductive mapping $F\left(x_{j}\right)$ is a monotonously increasing function of the input. The interval arithmetic presented by Moore [4] is used together with the extension principle on several membership $\alpha$ cuts of the fuzzy number $x_{j}$ for evaluating fuzzy expressions $[5,30,31]$. The fuzzy sets can be modified by intensifying or weakening modifiers [8].

Membership degree for fuzzy equality of two fuzzy sets can obtained by

$$
\mu_{A=B}=\sup _{x} \min \left[\mu_{A}(x), \mu_{B}(x)\right] .
$$

The calculation of inequalities is based on comparing modified identities. Fuzzy equalities and inequalities $<, \leq,=$, $\geq$ and $>$ produce new facts for rule-based fuzzy set systems.

Calculations with fuzzy numbers expand step by step the uncertainty of the results. Therefore, associations are important in reducing the uncertainty of calculations. $L E$ models are used for representing various types of associations, see Section 2.3.3. For example, high flow is associated with short time delay. 


\subsection{Parametric models}

Case-based systems can be built by integrating local parametric models. Linear parameter varying models (LPV) and fuzzy set systems are alternatives for working point models.

\subsubsection{Statistical models}

Statistical modelling in its basic form uses linear regression for solving coefficients for linear functions $F(\vec{x})$. Interactions between variables become important in the multiple regression. Principal component analysis (PCA) reduces the number of dimensions [32]. Latent structures and collinear variables are important in avoiding overfitting [33].

In the response surface methodology (RSM), the relationships are represented with multiple input, single output (MISO) models, which contain linear, quadratic and interactive terms [34]. Model selection algorithms are important in finding efficient predictions with less parameters [35]. Expert knowledge and robust fitting techniques can improve these models [36].

$L P V$ models extend application areas of the linear modelling. Semi-physical models bring appropriate calculated variables as inputs, see [37]. Fuzzy inputs move the analysis to several $\alpha$-levels. The interval arithmetic can be used within models and also the coefficients can be fuzzy numbers.

Various advanced methodologies are available for extending the statistical model approach. However, the high complexity makes the adaptation of the system difficult.

\subsubsection{Neural and neurofuzzy systems}

Artificial neural networks (ANN) are used as behavioural input-output models consisting of neurons. The response of each neuron $i$ is obtained by an activation function, $F_{i}$ :

$$
y_{i}=F_{i}\left(\sum_{j=1}^{m} w_{i j}^{N N} p_{j}+b_{i}^{N N}\right),
$$

whose input is calculated from a weighted sum of the normalised variables and a bias term: $w_{i j}^{N N}$ is the weight factor of the element $p_{j}$ in the input vector of the neuron $i$, and $b_{i}^{N N}$ a scalar bias. For the input layer, the elements $p_{j}$ are usually normalised values of the variables $x_{j}, j=$ $1,2, \ldots, m$. Network architectures differ from each other in their way of forming the net input, use of activation functions $F_{i}$ and number of layers.
Neurofuzzy systems use fuzzy neurons to combine the weight factors $w_{i j}$ and the inputs $p_{j}$ in (9). The activation function is handled with the extension principle from the fuzzy input, which is obtained by the fuzzy arithmetics. Different combinations with fuzzy and crisp weight factors and elements can be used in these models [38]. Cascade architectures of fuzzy set systems and neural networks are often called neurofuzzy systems. Neural computation is used for tuning fuzzy set systems which can be represented by neural networks, see [39].

Overfitting is a real risk in neural computation, especially in deep learning. Although the weights could be fuzzy, it is difficult to introduce this idea in the learning algorithms. However, fuzzy inputs are possible and then the responses would be fuzzy. The solutions can be extended with the NSFs in the same way as in the rule-based fuzzy set systems, see Section 2.2.1.

\subsubsection{Linguistic equations}

Linguistic equation ( $L E)$ approach originates from fuzzy set systems [13]. The main idea is to make compact systems which take into account nonlinearities by using the nonlinear scaling (Section 2.1.2) together with linear equations [14]. Extensions to more complex statistical models could be used but considerable improvements are not achieved if the NSFs are on a reasonable level.

The $L E$ systems can be extended to fuzzy systems by using the NSFs for transforming fuzzy numbers to both directions between the real and scaled values (Section 2.1.2). The linear equations are processed by the fuzzy interval analysis and finally the resulting fuzzy numbers are scaled back to the real scale.

Associations are simple interactions, e.g. a positive association leads to extending fuzziness for a sum of two fuzzy numbers. This is the normal way used in fuzzy arithmetic. On the other hand, a negative association means that the same sum has much less uncertainty.

Locations for all membership functions or singletons can be defined for one variable in each equation of a $L E$ model (Figure 2). Pruning is used for combining membership functions which are close to each other.

Fuzzy set systems can be changed into $L E$ models by replacing linguistic labels with real numbers (Figure 2). Slight adjusting of these locations is usually needed, and NSFs are generated from adjusted locations and the center points of the corresponding MFs. The model surface is directly used for very small fuzzy set systems as there are too few relations for the parameter-based approach. [14] 
Table 2: Adaptation

\begin{tabular}{llll}
\hline $\begin{array}{l}\text { Adaptation } \\
\text { level }\end{array}$ & Fuzzy set systems & Linguistic equations & Application \\
\hline $\begin{array}{l}\text { Scaling } \\
\text { Shape }\end{array}$ & 1. All the MFs & 1. All the NSFs & \\
& 2. Modify individual MFs & $\begin{array}{l}\text { 2.1 Parameters of NSFs from generalised } \\
\text { norms }\end{array}$ & $\begin{array}{l}\text { Working point adaptation } \\
\text { Smooth changes }\end{array}$ \\
Interactions & 3. The rules & 3. Update generalised norms & Drastic changes \\
\end{tabular}

Linguistic Takagi-Sugeno (LTS) models were developed for improving the operation of TS models in the border areas of local models [40]. The linear models are replaced by LE models by using the NSFs. The LTS models are robust solutions for applications where the same variables can be used for defining operating areas and in the submodels. No special smoothing algorithms are needed.

The nonlinear scaling is the key part of the $L E$ models: compact basic models can be extended with the statistical, fuzzy and neural methodologies.

\section{Adaptation}

Uncertainty processing and temporal analysis are essential in assessing the need for adaptation. Recursive data analysis provides material for the detection of changes and finally, consistent systems facilitate the efficient adaptation. The recursive part focuses on the scaling and the interactions are updated only if needed (Table 2 ).

\subsection{Recursive data analysis}

The parameters of the NSFs can be recursively updated by using generalised norms with the defined orders. This can be done real-time: the norm values are updated by including new equal sized sub-blocks in the calculations [41] The norm for several samples is obtained as the norm of the norms of the individual samples [22, 42]. Outliers should be excluded, but the suspicious values may mean that the operating conditions are changing, i.e. the identity of the short long term NSFs is not sufficient [41]. The new set of parameters is checked and corrected if the monotonous increase is not achieved.

The new orders are obtained by using the generalised skewness for the data extended with the data collected from the new situation [20]. The same check of monotonocity is performed in this analysis.
The uncertainties can be analysed since the calculations are based on several sub-blocks which can have different data distributions. Levels, changes, trends and fluctuations can be followed for all five parameters of each scaling function (Figure 1). Individual MFs can be analysed by using only the corner points of the trapezoidal MFs.

\subsection{Detection of changes}

The temporal analysis of levels and features representing the increase, decrease, trends and fluctuations introduces information for detecting changes in operating conditions. An increasing number of correction requirements can be an indication of changing operating conditions. The parameters obtained from the recent data need to be compared with the active parameters. The comparison, which is based on fuzzy inequalities $<, \leq,=, \geq$ and $>$, provides uncertain facts for the fuzzy decision making.

The generalised statistical process control (GSPC) is an additional tool for detecting changes, anomalies and novelties in connection with the operation [43]. Warnings and alarms are generated by the control charts.

The detection focuses on the NSFs since the sets of the $M F s$ can be built from the NSFs. The analysis of the individual MFs is a special case of this.

\subsection{System adaptation}

Fuzzy set systems can be adapted on three levels: (1) scaling the sets of $M F s$, (2) adapting the shapes of the MFs and (3) adapting the rulebase. The scaling is easy and well understood, but the importance to understand correctly the meanings of the MFs is not so well shared. Adapting the rulebase should really be the last in the sequence (Table 2).

In the adapting of the NSFs, the first level is also the scaling: the functions become sharper or wider. Adapting the shape of the functions is the key and there are two lev- 
els of the operation: (2.1) new parameters are obtained by the generalised norms with active orders, and (2.2) the orders of the norms are re-analysed. The directions of interactions handled with linear equations provide results for the rules as well.

The interactions are re-analysed if the adaptation of the NSFs does not lead to sufficient improvements.

Genetic algorithms are well suitable for adapting the LE systems with a very high number of parameters. Efficient calculations can be achieved since the acceptable range $\left[\frac{1}{3}, 3\right]$ of the shape factors $\alpha_{j}^{-}$and $\alpha_{j}^{+}$accept only the solutions which are monotonously increasing. All the parameters can be handled simultaneously.

\section{Application areas}

The nonlinear scaling functions provide compact links between the applications and uncertainty processing. Uncertainty processing brings the data-driven development and solutions available to the assessment based on domain expertise.

\subsection{Modelling}

Case-based models can be combined as multimodel $L E$ systems, where a fuzzy decision module is used for proving the degree of membership for each active model. The decisions can be based on fuzzy working point models. Since the interactions are presented by linear equations, these models extend the linear parameter varying $(L P V)$ models. The submodels can also have totally different structures and variables. The LTS models are special cases of these models. [44]

\subsection{Intelligent control}

Intelligent methods are widely used for nonlinear multivariable control systems, e.g. a large number of highly successful fuzzy logic control (FLC) applications are implemented in process industry. The system structures are similar to the fuzzy models: crisp responses are obtained by defuzzification.

In feedback control, the $L E$ controllers use similar structures enhanced with the NSFs. Intelligent analysers are increasingly important in these systems [45]. Modelbased control is widely applied to industrial applications [46]. The $L E$ control systems use predefined model- based techniques and activate special features when needed, since the adaptation needs to be fast [47].

Fuzzy adaptation of em LE controllers opens additional possibilities to the decision making by extending the smart working point control. The operation is kept smooth by limiting the allowed temperature range in difficult situations. [48]

\subsection{Diagnostics}

Rule-based fuzzy set systems result fuzzy sets which include alternative faults with degrees of membership. Efficient solutions can be developed integrating condition monitoring data in the form of intelligent indices into the operation and maintenance. The NSFs methodologies are also used for handling performance measures used in the same scale as intelligent condition and stress indices. Performance indicators are responses of the process, machine or system to the stress contributions analyzed from process and condition monitoring data. The systems are adapted to the changing operation conditions by the recursive tuning of the NSFs. [49]

Fuzzy relational systems are based on intensities of interactions between symptoms and faults. Symptoms are facts, including labels, inequalities and trends. As the labels do not need to any links with numerical values, the relations can be the first or highest level in the diagnostics.

\section{Discussion}

In applications, the systems need to use both crisp and fuzzy numbers as well as additional facts. The domain expertise can be introduced by fuzzy labels and understanding of variable meanings. Fuzzy relations are used within higher level systems. Neural models are numeric models in the same way as the statistical and $L E$ models.

Nonlinear scaling and $L E$ systems with fuzzy arithmetic make the systems more compact. Rule-based fuzzy systems and neural networks can be simplified by including layers which use NSFs. The coefficients of the $L E$ systems are based on linear regression. Therefore, feedforward $L E$ models can be transformed to feedforward controllers. Adaptation can be done even recursively with the NSFs. The overall solution forms a feasible basis for building tunable large-scale systems.

The NSFs and $L E$ models are compact and easy to adapt but the structural limitations need to be kept in mind. The basic systems are feasible for monotonously in- 
creasing subsystems where the $L E$ models and fuzzy systems can be transformed to both directions. Higher levels need fuzzy systems for utilizing contradictory data, non-numeric information and balancing different operating conditions.

\section{Conclusions}

Meanings of variables are the key to handling expertise and uncertainty processing in automation applications. Nonlinear scaling provides additional calculation levels which simplify statistical, fuzzy and neural solutions. Large-scale applications can be built and tuned as modular parametric systems. The embedded uncertainty processing in the recursive analysis form a basis for the adaptation. Fuzzy systems are needed for balancing contradictory information, non-numeric expertise and operating areas.

\section{References}

[1] Zadeh LA. Fuzzy sets. Inf Control. 1965;8(June):338-53.

[2] Zimmermann HJ. Fuzzy set theory and its applications. Kluwer Academic Publishers; 1992.

[3] Pedrycz W. An identification algorithm in fuzzy relational systems. Fuzzy Sets Syst. 1984;13(2):153-67.

[4] Moore RE. Interval Analysis. Englewood Cliffs (N)): Prentice Hall; 1966.

[5] Buckley JJ, Feuring T. Universal approximators for fuzzy functions. Fuzzy Sets Syst. 2000;113(3):411-5.

[6] Jantzen J, Poulsen NK. Adaptation in the Fuzzy Self-Organizing Controller. In: Proceedings of Eunite 2003 - European Symposium on Intelligent Technologies, Hybrid Systems and their implementation on Smart Adaptive Systems, July 10-11, 2003, Oulu, Finland. Aachen: Wissenschaftsverlag Mainz; 2003. p. 49-57.

[7] Pirrello L, Yliniemi L, Leiviskä K, Galluzzo M. Self-tuning fuzzy control of a rotary dryer. In: Basanez L, de la Puente JA, editors. Proceedings of the 15th Triennial World Congress, Barcelona, Spain, July 21-26, 2011. IFAC; 2002. p. 125-130. http://www.ifacpapersonline.net/

[8] De Cock M, Kerre EE. Fuzzy modifiers based on fuzzy relations. Inf Sci. 2004;160(1-4):173-99.

[9] Schmidhuber J. Deep learning in neural networks: an overview. Neural Netw. 2015 Jan;61 Supplement C:85-117. Available from: http://www.sciencedirect.com/science/article/pii/S089360801 4002135

[10] Kohonen T. Self-Organizing Maps. Berlin: Springer; 1995. https://doi.org/10.1007/978-3-642-97610-0.

[11] Barreto GA. Lime kiln hybrid control system. In: Proceedings of Dynamic Modeling Control Applications for Industry Workshop, Vancouver. IEEE Industry Applications Society; 1997. p. 44-50.
[12] Bezdek JC, Tsao EC, Pal NR. Fuzzy Kohonen clustering networks. In: [1992 Proceedings] IEEE International Conference on Fuzzy Systems; 1992. p. 1035-1043.

[13] Juuso EK, Leiviskä K. Adaptive Expert Systems for Metallurgical Processes. IFAC Proceedings Volumes. 1992;25(17):119-124. https://doi.org/10.1016/B978-0-08-041704-2.50027-3.

[14] Juuso EK. Integration of Intelligent Systems in Development of Smart Adaptive Systems. Int J Approx Reason. 2004;35(3):30737.

[15] Juuso EK. Tuning of Large-Scale Linguistic Equation (LE) Models with Genetic Algorithms. In: Kolehmainen M, editor. Revised selected papers of the International Conference on Adaptive and Natural Computing Algorithms - ICANNGA 2009, Kuopio, Finland, Lecture Notes in Computer Science. vol. LNCS 5495. Heidelberg: Springer-Verlag; 2009. p. 161-170.

[16] Juuso EK. Recursive Tuning of Intelligent Controllers of Solar Collector Fields in Changing Operating Conditions. IFAC Proceedings Volumes. 2011;44(1):12282-12288. https://doi.org/ 10.3182/20110828-6-IT-1002.03621.

[17] Juuso EK. Smart Adaptive Big Data Analysis with Advanced Deep Learning. Open Eng. 2018;8(1):403-16. Available from: www.scopus.com

[18] Juuso EK. Informative process monitoring with a natural language interface. In: 2016 UKSim-AMSS 18th International Conference on Modelling and Simulation, 6-8 April, 2016, Cambridge, UK. IEEE Computer Society; 2016. p. 105-110.

[19] Juuso EK. Linguistic Equation Framework for Adaptive Expert Systems. In: Stephenson J, editor. Modelling and Simulation 1992, Proceedings of the 1992 European Simulation Multiconference, York, UK, June 1-3, 1992. San Diego, USA: SCS International; 1992. p. 99-103.

[20] Juuso E, Lahdelma S. Intelligent scaling of features in fault diagnosis. In: 7th International Conference on Condition Monitoring and Machinery Failure Prevention Technologies, CM 2010 - MFPT 2010, 22-24 June 2010. Volume 2. Stratford-upon-Avon, UK; 2010. pp. 1358-72. Available from www.scopus.com

[21] Juuso EK. Integration of Knowledge-based Information in Intelligent Condition Monitoring. In: 9th International Conference on Condition Monitoring and Machinery Failure Prevention Technologies, 12-14 June 2012, London, UK. Volume 1. NY, USA: Curran Associates; 2012. pp. 217-28., Available from www.scopus.com

[22] Lahdelma S, Juuso E. Signal processing and feature extraction by using real order derivatives and generalised norms. Part 1: methodology. International Journal of Condition Monitoring. 2011;1(2):46-53.

[23] Dubois D, Prade H, Ughetto L. Fuzzy Logic, Control Engineering and Artificial Intelligence. In: Verbruggen HB, Zimmermann HJ, Babuska R, editors. Fuzzy Algorithms for Control, International Series in Intelligent Technologies. Boston: Kluwer; 1999. pp. 1757.

[24] Takagi T, Sugeno M. Fuzzy identification of systems and its applications to modeling and control. IEEE Trans Syst Man Cybern. 1985;15(1):116-32.

[25] Babuška R. Fuzzy Modeling and Identification. Boston: Kluwer Academic Publisher; 1998. p. 100.

[26] Johansen TA, Babuška R. Multiobjective identification of TakagiSugeno fuzzy models. IEEE Trans Fuzzy Syst. 2003;11(6):847-60.

[27] Mendel JM. Advances in type-2 fuzzy sets and systems. Information Sciences. 2007;177(1):84-110. 105 https://doi.org/10. 1016/j.ins.2006.05.003. 
[28] Krone A, Kiendl H. Automatic Generation of Positive and Negative Rules for Two-Way Fuzzy Controllers. In: Zimmermann HJ, editor. Proceedings of the Second European Congress on Intelligent Technologies and Soft Computing -EUFIT'94, Aachen, September 21 - 23, 1994. vol. 1. Aachen: Augustinus Buchhandlung; 1994. p. 438-447.

[29] Krone A, Schwane U. Generating Fuzzy Rules from Contradictory Data of Different Control Strategies and Control Performances. In: Proceedings of the Fuzz-IEEE'96, New Orleans, USA; 1996. p. 492-497.

[30] Buckley JJ, Qu Y. On using $\alpha$-cuts to evaluate fuzzy equations. Fuzzy Sets Syst. 1990;38(3):309-12.

[31] Buckley JJ, Hayashi Y. Can neural nets be universal approximators for fuzzy functions? Fuzzy Sets Syst. 1999;101(3):323-30.

[32] Jolliffe IT. Principal Component Analysis. 2nd ed. New York: Springer; 2002. 487 pp.

[33] Gerlach RW, Kowalski BR, Wold HO. Partial least squares modelling with latent variables. Anal Chim Acta. 1979;112(4):417-21.

[34] Box GE, Wilson KB. On the experimental attainment of optimum conditions. J R Stat Soc B. 1951;13(1):1-45.

[35] Efron B, Hastie T, Johnstone I, Tibshirani R. Least angle regression. Ann Stat. 2004;32(2):407-99.

[36] Morgenthaler S, Schumacher MM. Robust analysis of a response surface design. Chemom Intell Lab Syst. 1999;47(1):127-41.

[37] Ljung L. System Identification - Theory for the User. 2nd ed. Upper Saddle River (N.J.): Prentice Hall; 1999.

[38] Fullér R. Introduction to Neuro-Fuzzy Systems. Advances in Soft Computing. Springer; 2000. 289 pp. https://doi.org/10. 1007/978-3-7908-1852-9.

[39] Jang JS. ANFIS: Adaptive-Network-based Fuzzy Inference Systems. IEEE Trans Syst Man Cybern. 1993;23(3):665-85.

[40] Juuso EK. Development of Multiple Linguistic Equation Models with Takagi-Sugeno Type Fuzzy Models. In: Carvalho JP, Dubois D, Kaymak U, Sousa JC, editors. Proceedings of 2009 IFSA World 35 Congress / 2009 EUSFLAT Conference. Lisboa, Portugal: IFSA-EUSFLAT; July 20-24, 2009. pp. 1779-84. http://www.eus flat.org/publications_proceedings_IFSA-EUSFLAT_2009.php
[41] Juuso EK. Recursive Data Analysis in Large Scale Complex Systems. In: Juuso E, Dahlquist E, Leiviskä, editors. Proceedings of The 9th EUROSIM Congress on Modelling and Simulation, EUROSIM 2016, The 57th SIMS Conference on Simulation and Modelling SIMS 2016. No. 142 in Linköping Electronic Conference Proceedings. Linköping University Electronic Press; 2018. p. 1053-1059. https://doi.org/10.3384/ecp171421053.

[42] Lahdelma S, Juuso E. Signal processing and feature extraction by using real order derivatives and generalised norms. Part 2: applications. International Journal of Condition Monitoring. 2011;1(2):54-66.

[43] Juuso EK. Generalised statistical process control GSPC in stress monitoring. IFAC-PapersOnLine. 2015;48(17):207-12. Available from: www.scopus.com

[44] Juuso EK. Intelligent Multimodel Simulation in Decomposed Systems. In: Øi LE, Komulainen T, Bye RT, Nord LO, editors. Proceedings of The 59th Conference on Simulation and Modelling (SIMS 59), 26-28 September 2018, Oslo Metropolitan University, Norway. No. 153 in Linköping Electronic Conference Proceedings. Linköping University Electronic Press; 2018. p. 308-315.

[45] Juuso EK. Intelligent trend analysis for a solar thermal energy collector field. IOP Conf Ser Earth Environ Sci. 2018;136:1-8.

[46] Camacho EF, Bordons C. Model Predictive Control in the Process Industry. Berlin, Germany: Springer; 1995. https://doi.org/ 10.1007/978-1-4471-3008-6.

[47] Juuso EK, Yebra LJ. Model-based intelligent control of a solar energy collector field. In: Proceedings - 8th EUROSIM Congress on Modelling and Simulation, EUROSIM 2013, 10-13 September, 2013, Cardiff, UK; 2013. p. 513-518. Available from: www.scopus.com https://doi.org/10.1109/EUROSIM.2013.92.

[48] Juuso EK. Fuzzy adaptation of intelligent control for solar thermal power plants. In: 30th European Modeling and Simulation Symposium. EMSS; 2018. pp. 343-8., Available from www.scopus.com

[49] Juuso EK. Intelligent Performance Analysis with a Natural Language Interface. Management Systems in Production Engineering. 2017;25(3):168-75. 